then you miss out on a mind of Mayr's calibre and influence.

Mayr has now published a major ornithological monograph and a popular book on evolution in the past year. I sense he is only just getting up to speed. My next request is for an autobiography.

Mark Ridley is in the Department of Zoology, University of Oxford, South Parks Road, Oxford OX1 3PS, UK.

\section{Tuning in to radio galaxies}

The Physics of Extragalactic Radio Sources

by David S. De Young

University of Chicago Press: 2002. 450 pp. $\$ 45$

\section{K. I. Kellermann}

After the Second World War, scientists, especially in England and Australia, turned their wartime radar equipment towards the skies to follow up on the pre-war discovery by Karl Jansky and Grote Reber of radio emission from the Milky Way. Although hampered at first by poor angular resolution and limited sensitivity, they were able to detect powerful sources of non-thermal radio emission from several distant galaxies. During the 1950s and 1960s, powerful interferometric and aperture-synthesis techniques were developed. When combined with theoretical advances, primarily in the Soviet Union, they showed that the observed radio emission was due to synchrotron radiation emitted from extensive clouds of ultrarelativistic electrons moving in weak magnetic fields far removed from the parent galaxy. The energy required in the form of relativistic particles and magnetic fields appeared to be enormous.

But some extragalactic radio sources were found to be very much smaller and surprisingly appeared to vary in intensity on timescales of months or less. Interferometers and lunar occultations were used to locate radio sources accurately in the sky, leading to observations of ever more distant radio galaxies and to the discovery of quasars. By the late 1960s, tape-recording interferometers had stretched radio-interferometer baselines to intercontinental dimensions. With angular resolutions better than 0.001 arcseconds available, radio astronomers were able to show that the source of energy that powers radio galaxies and quasars was as small as a few light years across or less.

Today, radio telescopes are a million times more sensitive than the pioneering post-war instruments, and more than a million extragalactic radio sources have been catalogued. Radio astronomers use wavelengths ranging from less than a millimetre to several metres, which is comparable to the range from the

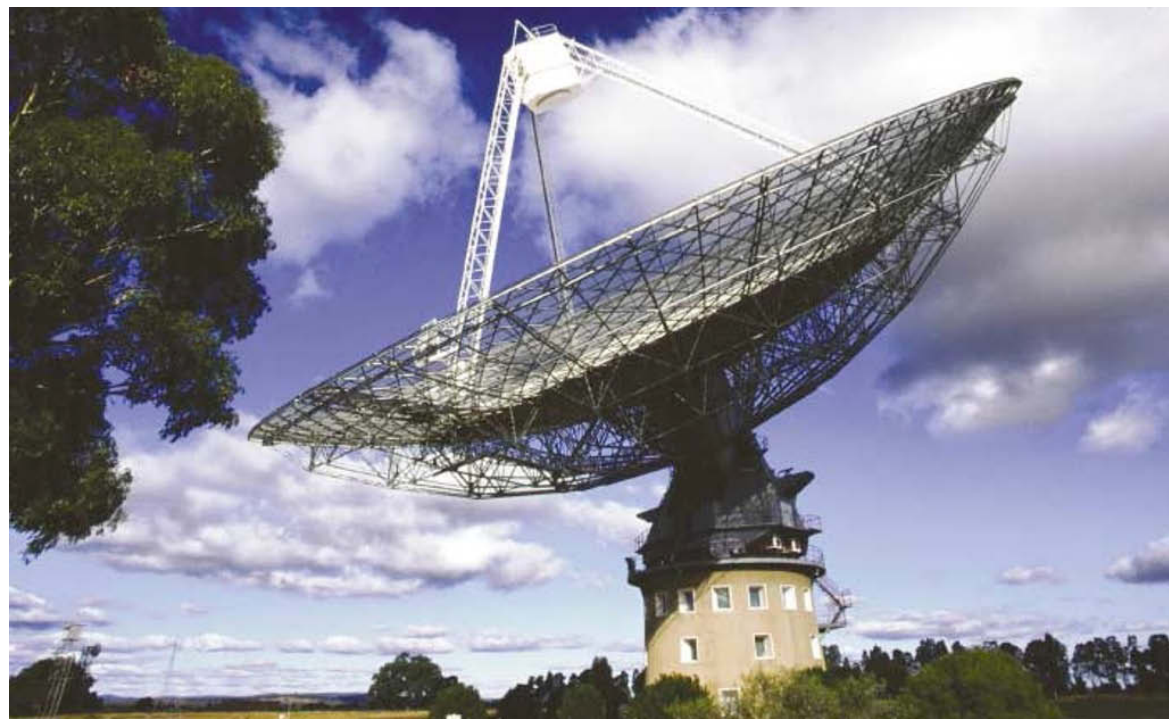

Radio star: the Parkes Radio Telescope near Alectown in Australia detects extragalactic signals.

infrared through the optical and ultraviolet to the X-ray parts of the electromagnetic spectrum. Huge radio-telescope arrays show remarkably fine detail in the synchrotron clouds, revealing thin jets that carry the relativistic electron gas from active galactic nuclei (AGN) out to distant radio lobes, as much as a million light years or more away from the central engine. Global radio-interferometer observations using angular resolutions up to 100 times better than that of the Hubble Space Telescope show directly the ejection of relativistic plasma from the central engine with apparent velocities in excess of the speed of light. This is an illusion caused by the finite signal-propagation time from a relativistically moving source of radiation.

However, the new observations may have raised more questions than they answer. The source of energy is still unclear, although it is widely assumed to be associated with accretion of material into a huge black hole up to $10^{9}$ times as massive as the Sun. How the energy is converted into a highly collimated beam of relativistic particles remains a mystery. Why are some galaxies powerful radio sources, whereas others are not? Why are powerful quasars sometimes radio-loud and sometimes radio-quiet? To what extent is the wide range of observed extragalactic radio-source properties intrinsic, and how much is due to geometrical effects? What is the relationship between quasars, AGN and galaxy formation, especially the burst of star formation often seen in distant galaxies?

In The Physics of Extragalactic Radio Sources De Young discusses all of these issues, starting with a qualitative review of the properties of radio galaxies and quasars. Other chapters discuss the application of synchrotron radiation, plasma and jet physics relevant to extragalactic radio sources. Classical formulae are derived only where the author has a new approach to the derivation that leads to a better understanding of the physics involved. In other cases, the reader is referred to the appropriate literature. In the later chapters De Young applies the theory to both extended and compact radio sources, relativistic jets and AGN, including a discussion of unified radio-source models which attempt to explain the range of observed properties as the result of the orientation of the relativistic jet or an obscuring torus surrounding the central engine. He discusses the nature of the host galaxy or associated quasar and includes a review of their optical and $\mathrm{X}$-ray properties.

There are few original drawings. Most of the illustrations are taken from the literature, but are sometimes out of date in this rapidly moving field and often have inadequate captions or explanation. There are a few errors, some of which seem to have been introduced by the editors. Nevertheless, the book will be a valuable resource for all astrophysics students and to everyone working in the field of extragalactic astronomy. As the author points out in the preface, no other book is devoted to this important field of astrophysics. K. I. Kellermann is at the National Radio Astronomy Observatory, Edgemont Road, Virginia 22903, USA.

\section{Exactly the same but different}

\section{The Secret}

by Eva Hoffman

Secker \& Warburg: 2001. 263 pages. $£ 15.99$

Justine Burley

One frequently voiced objection to human cloning is that clones would not have the same opportunities for individual development as children produced by sexual reproduction (see, for example, Nature 412, 
583; 2001). In The Secret, Eva Hoffman's first work of fiction, she probes the psychological impact on a cloned child, and on others around her, of the knowledge of her origins.

The story is narrated by Iris, the cloned daughter of a beautiful, highly successful woman (the cell donor) who has retreated to a leafy midwestern US suburb to bring up her child away from negative influences and prying eyes. Iris's cloistered childhood is characterized by a deep sense of being at one with her mother - this feels natural to both but discomfits everyone else. It is also marked by The Weirdness, or ill-defined selfknowledge, and The Strange Look, which Iris detects in others, including her mother. Her mother's lover, who was unable to discover the secret of the household, leaves when Iris is in her teens, intensifying her curiosity about her 'real father'. She snoops and discovers that she is a clone.

Iris's now rational awareness of her beginnings pitches her into an emotional void and, after violently rejecting her mother, she sets off in search of her self. Iris's sense of anomie intensifies when relatives from whom she craves love and acceptance are unable to respond to this identical copy of their sister/daughter. Desperate yet defiant, Iris sleeps with her mother's ex-lover. She does ultimately carve out a life for herself but it is devoid of family contact - her identity has been won at great cost.

The message that will be drawn from The Secret, whether or not it was intended by Hoffman, is that cloning has adverse, acute and disturbing effects on the clone's personal development and on family and other social relationships, and should therefore be banned.

But would all instances of human cloning entail deleterious psychological burdens and dysfunctional relationships of the kind described by the author? If Iris had been provided with information in childhood about how she was conceived, would learning that she is a clone be so traumatic? Might not a clone instead feel special in the same way that children born by in vitro fertilization reportedly do? If Iris's mother had not been so overprotective, would her child have felt less isolated from, and less different to, her peers? If her extended family had not been opposed to cloning, could she not have had satisfying family relationships in which her own personality was recognizable from early infancy and in which her physiological similarity to her mother preoccupied others less? Do we not have good reason to be suspicious of any man who cannot adequately distinguish emotionally and sexually between his ex-partner and her daughter, whatever 'sameness' exists between the two women? The answers invited by these questions suggest that Iris's experience was not necessarily an inevitable consequence of her cloning origin.

Even if we assume for the sake of argument that all clones would be likely to face the kind of struggles that Iris does in The Secret, is this enough of a reason to ban human cloning? The answer has to be no. If the reasonable prediction of psychological damage, the compromise of autonomy and fraught relationships is weighty enough to support a ban on asexual reproduction, then it must also be weighty enough to support a ban on sexual reproduction. It is mistaken to say that cloning involves harm of a different magnitude, because it can be seen that the welfare of children born by natural procreation is compromised severely and permanently by various behaviours of their parents, as well as by societal attitudes. It may be that no one should be allowed to procreate when it is likely that their offspring will suffer welfare-related harms of the kind discussed. But few people are prepared to stand up and argue this.

Hoffman uses the vehicle of fiction to explore a serious topic. Her strengths as an author lie in her ability to describe dislocation, alienation and individuality against different backdrops; see, for example, her much-acclaimed autobiography, Lost in Translation, in which, as a Polish emigrant, she struggles to find a sense of self and place in the New World. However, readers of The Secret should exercise caution if they were hoping for a comprehensive psychosocial commentary on the impact of cloning.

Justine Burley is at Exeter College, University of Oxford, Oxford OX1 3DP, UK.

\section{Science in culture}

\section{Cosmic opera}

Poussières d'Etoiles (Stardust), a multisensory experience in Paris.

Alison Abbott

Technology and imagination act symbiotically in the 'cosmic opera' Poussières d'Etoiles

(Stardust). Conceived by Philippe Corbin and designed for the cavernous hall of the Cité des Sciences on the outskirts of Paris, it addresses the senses of sight, hearing and smell. With few words, the three-act, anti-anthropocentric show tells us that we are made up of atoms formed in stars that may have died before our Sun was born.

Spectators walk into a complex light show that takes up all 50,000 cubic metres of the hall, and are greeted by a smell of damp earth and sounds that move from pure frequencies to scratching, chattering electronic music. Light images of immense butterflies take wing. The situation is intended to place the spectators outside their normal dimensions and prepare them for their virtual journey from the Big Bang to the creation of life on Earth.

The second act begins with the activation of escalators that carry spectators to luminous sofas on the mezzanine level. Here they lie and

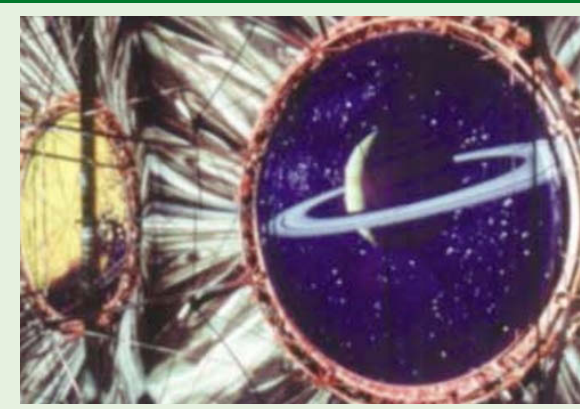

Eye of the beholder: the second act of Poussières d'Etoiles shows the development of the Universe.

watch, for 30 absorbing minutes, a breathtaking series of images projected onto the 2,400square-metre ceiling. Many of the images are from the Hubble Space Telescope. Astronomers may recognize the Horsehead nebula, the Hubble Towers (interstellar clouds) and the Pleiades star cluster, which go by without comment as the Universe develops.

The music, like the Universe, becomes more structured, eventually taking the form of an operatic libretto, although without significant text. The composer, Nicolas Frize, says that the celestial bodies have neither soul nor feeling, so literal dialogue in a libretto would be out of place. For the final act, after the Earth has made its first appearance in the virtual heavens, the voices of the choir evolve into drops of water, then torrential rain. The spectators look down the escalator and see the hall submerging in virtual floods as the Earth's primeval oceans fill. They descend to watch the first organic molecules appearing in the oceans, then see life emerging in all its forms. The smell of perfumed humidity, like a sauna, pervades.

The glory of the show lies in the light-handed presentation of the beauty of astronomy and cosmology, which avoids didacticism. Frize's intelligent, evocative and moving music adds fundamentally to the poetry of the second act. But the show's weakness is its clumsy choreography of spectators, who are herded around like confused package tourists, often understanding what they should be experiencing only after the moment has passed.

Alison Abbott is Nature's Senior European Correspondent.

Poussières d'Etoiles runs nightly, from Tuesday to Saturday, at the Cité des Sciences in Paris until 2004. 